\title{
Collider Tests for Neutrino Mass Generation in a Supersymmetric World with $R$-Parity Violation
}

\author{
M. B. Magro \\ Instituto de Física, Universidade de São Paulo, CP 66318, 05389-070, São Paulo-SP, Brazil.
}

Received on 18 February, 2003.

\begin{abstract}
We summarize the low energy features of a supersymmetric class of models with bilinear $R$-parity violation. We analyze two cases where the supersymmetry breaking is mediated either by supergravity or by anomaly induced contributions to the soft parameters and compare both scenarios in the context of recent neutrino conversion data and collider physics. We show that both classes of models have a large potential to discoveries in collider experiments as well as in neutrino experiments.
\end{abstract}

\section{Introduction}

Supersymmetry (SUSY) is a promising candidate for physics beyond the Standard Model (SM). The search for supersymmetric partners of the SM particles constitutes an important goal of current high energy colliders like the Tevatron, and future colliders like the LHC or a linear $e^{+} e^{-}$collider. However, no positive signal has been observed so far. Therefore, if supersymmetry is a symmetry of nature, it is an experimental fact that it must be broken. The three best known classes of models for supersymmetry breaking are gravity-mediated (SUGRA) [1], where SUSY is assumed to be broken in a hidden sector by fields which interact with the visible particles only via gravitational interactions; gaugemediated [2], where SUSY is broken in a hidden sector and transmitted to the visible sector via SM gauge interactions of messenger particles; and anomaly-mediated supersymmetry breaking (AMSB) [3], which is based on the observation that the super-Weyl anomaly gives rise to loop contribution to sparticle masses. In this work we will concentrate our searches in SUGRA and AMSB prescriptions.

So far most of the effort in searching for supersymmetric signatures has been confined to the framework of $R$-parity $\left(R_{P}\right)$ conserving realizations; however recent data on solar and atmospheric neutrinos give a robust evidence for neutrino conversions [4], probably the most profound discovery in particle physics in the recent years. It has been suggested long ago that neutrino masses and supersymmetry may be deeply tied together $[5,6]$. Indeed, SUSY models exhibiting $R$-parity violation can lead to neutrino masses and mixings [6] in agreement with the current solar and atmospheric neutrino data.

The high statistics data by the SuperKamiokande collaboration [4] has confirmed the deficit of atmospheric muon neutrinos, especially at small zenith angles, providing a strong hint for neutrino conversion. Although massless neutrino conversions [7] can be sizeable in matter, it is fair to say that simplest interpretation of the present data is in terms of massive neutrino oscillations. The atmospheric data indicate $\nu_{\mu}$ to $\nu_{\tau}$ flavor oscillations with maximal mixing [8], while the solar data can be accounted for in terms of either small (SMA) and large (LMA) mixing MSW solutions [9], as well as through vacuum or just-so solutions [10]. A large mixing among $\nu_{\tau}$ and $\nu_{e}$ is excluded both by the atmospheric data and by reactor data on neutrino oscillations [11].

It has been recently shown [6] that one can explain the neutrino data through SUGRA models with bilinear $R$ parity violation $(\mathrm{BRpV})$. The attainable range of the squared difference of the neutrino masses, $\Delta m_{i j}^{2}, i, j$ standing for the neutrino flavors, favors the interpretation of the atmospheric neutrino data. It was possible to fix the atmospheric angle and at the same time obey the $\mathrm{CHOOZ}$ constraint. For the solar angle, however, the results depend on whether one wants to work in a SUGRA motivated scenario or not. For the SUGRA scenario it was shown that this $R$-parity violating model allows only the small mixing and MSW solution (SMA), while for the minimal supersymmetric case (MSSM) also LMA and vacuum oscillation solutions are possible. Under the assumption of SUGRA conditions the atmospheric scale is calculable by the renormalization group evolution due to the non-zero bottom quark Yukawa coupling. In this case one predicts the small mixing angle (SMA) MSW solution to be the only viable solution to the solar neutrino problem. In contrast, for the general MSSM model, where the above assumptions are relaxed, on can implement a bi-maximal [12] neutrino mixing scheme, in which the solar neutrino problem is accounted for through large mixing angle solutions, either MSW or just-so.

It is interesting to notice that neutrino mass models based on $R$-parity violation can be tested at colliders [13, 14]. In this work, we summarize two different approaches to $R$-parity violating models that can be tested either at colliders or neutrino data. The first is based on AMSB supersymmetry braking and the second on SUGRA. In the latter we show that is possible to search for $R$-parity violation SUSY via the production of multileptons $(\geq 3 \ell)$ at the Fermilab Tevatron within the framework of the simplest supergravity (SUGRA) model without $R$-parity [15]. In the first we sum- 
marize the main features of a new realization of $R$-parity violating SUSY models with anomaly-mediated supersymmetry braking.

We consider a supersymmetrical model that includes the following bilinear terms in the superpotential [15]

$$
W_{B R p V}=W_{M S S M}-\varepsilon_{a b} \epsilon_{i} \hat{L}_{i} \hat{H}_{i}^{b},
$$

where the last term violates $R$-parity. In order to reproduce the values of neutrino masses indicated by current data [16] we choose the parameter space where $\left|\epsilon_{i}\right| \ll|\mu|[6]$. The relevant bilinear terms in the soft supersymmetry breaking sector are

$$
\begin{aligned}
V_{\text {soft }}= & m_{H_{u}}^{2} H_{u}^{a *} H_{u}^{a}+m_{H_{d}}^{2} H_{d}^{a *} H_{d}^{a}+M_{L_{i}}^{2} \tilde{L}_{i}^{a *} \tilde{L}_{i}^{a} \\
& -\varepsilon_{a b}\left(B \mu H_{d}^{a} H_{u}^{b}+B_{i} \epsilon_{i} \tilde{L}_{i}^{a} H_{u}^{b}\right)
\end{aligned}
$$

where the terms proportional to $B_{i}$ are the ones that violate $R$-parity. The explicit $R$-parity violating terms induce vacuum expectation values (vev) $v_{i}, i=1,2,3$ for the sneutrinos, in addition to the two Higgs vev's $v_{u}$ and $v_{d}$.

This paper is organized as follows. We summarize in Section 2 the main features of a bilinear $R$-parity violating SUSY model with anomaly-mediated SUSY breaking. This section also contains an overview of the supersymmetric spectrum in this model as well as the properties of the $\mathrm{CP}$-odd, CP-even and charged scalar particles, concentrating on the mixing angles that arise from the introduction of the $R$-parity violating terms. Section 3 contains a phenomenological study of the production of multileptons at the Fermilab Tevatron within the framework of a simplest SUGRA model without $R$-parity. We look at the capabilities of the RUN II at the Tevatron probe bilinear $R$-parity violation. Finally, in Section 4 we draw our final remarks on the models showed here.

\section{The AMSB-BRpV Model}

In AMSB models, the soft terms are fixed in a nonuniversal way at the unification scale which we assumed to be $M_{G U T}=2.4 \times 10^{16} \mathrm{GeV}$. We considered the running of the masses and couplings to the electroweak scale, assumed to be the top mass, using the one-loop renormalization group equations (RGE) [17]. In the evaluation of the gaugino masses, we included the next-to-leading order (NLO) corrections coming from $\alpha_{s}$, the two-loop top Yukawa contributions to the beta-functions, and threshold corrections enhanced by large logarithms; for details see [18]. The NLO corrections are especially important for the gaugino mass $M_{2}$, leading to a change in the wino mass by more than $20 \%$.

One of the virtues of AMSB models is that the $S U(2) \otimes$ $U(1)$ symmetry is broken radiatively by the running of the RGE from the GUT scale to the weak one. This feature is preserved by our model since the one-loop RGE are not affected by the bilinear $R_{P}$ violating interactions [17]. In this work, we made the simplest assumption that $R$-parity is violated only in the third generation. A full three generation model in under preparation. Therefore, in our model, the electroweak symmetry is broken by the vacuum expectation values of the two Higgs doublets $H_{d}$ and $H_{u}$, and the neutral component of the third left slepton doublet $\widetilde{L}_{3}$. We denote these fields as

$$
\begin{aligned}
& H_{d}=\left(\begin{array}{c}
\frac{1}{\sqrt{2}}\left[\chi_{d}^{0}+v_{d}+i \varphi_{d}^{0}\right] \\
H_{d}^{-}
\end{array}\right), \\
& H_{u}^{+}=\left(\begin{array}{c}
\frac{1}{\sqrt{2}}\left[\chi_{u}^{0}+v_{u}+i \varphi_{u}^{0}\right]
\end{array}\right), \\
& \widetilde{L}_{3}=\left(\begin{array}{c}
\frac{1}{\sqrt{2}}\left[\tilde{\nu}_{\tau}^{R}+v_{3}+i \tilde{\nu}_{\tau}^{i 0}\right] \\
\tilde{\tau}^{-}
\end{array}\right) .
\end{aligned}
$$

The above vev's $v_{i}$ can be obtained through the minimization conditions, or tadpole equations, which in the AMSB-BRpV model are

$$
\begin{aligned}
t_{d}^{0}= & \left(m_{H_{d}}^{2}+\mu^{2}\right) v_{d}-B \mu v_{u}-\mu \epsilon_{3} v_{3}+ \\
& \frac{1}{8}\left(g^{2}+g^{\prime 2}\right) v_{d}\left(v_{d}^{2}-v_{u}^{2}+v_{3}^{2}\right) \\
t_{u}^{0}= & \left(m_{H_{u}}^{2}+\mu^{2}+\epsilon_{3}^{2}\right) v_{u}-B \mu v_{d}+B_{3} \epsilon_{3} v_{3}- \\
& \frac{1}{8}\left(g^{2}+g^{\prime 2}\right) v_{u}\left(v_{d}^{2}-v_{u}^{2}+v_{3}^{2}\right) \\
t_{3}^{0}= & \left(m_{L_{3}}^{2}+\epsilon_{3}^{2}\right) v_{3}-\mu \epsilon_{3} v_{d}+B_{3} \epsilon_{3} v_{u}+ \\
& \frac{1}{8}\left(g^{2}+g^{\prime 2}\right) v_{3}\left(v_{d}^{2}-v_{u}^{2}+v_{3}^{2}\right)
\end{aligned}
$$

at tree level. At the minimum we must impose $t_{d}^{0}=t_{u}^{0}=$ $t_{3}^{0}=0$. In practice, the input parameters are the soft masses $m_{H_{d}}, m_{H_{u}}$, and $m_{L_{3}}$, the vev's $v_{u}, v_{d}$, and $v_{3}$ (obtained from $m_{Z}, \tan \beta$, and $m_{\nu_{\tau}}$ ), and $\epsilon_{3}$. We then use the tadpole equations to determine $B, B_{3}$, and $|\mu|$.

One-loop corrections to the tadpole equations change the value of $|\mu|$ by $\mathcal{O}(20 \%)$, therefore, we also included the one-loop corrections due to third generation of quarks and squarks [15]:

$$
t_{i}=t_{i}^{0}+\tilde{T}_{i}(Q)
$$

where $t_{i}$, with $i=d, u$, are the renormalized tadpoles, $t_{i}^{0}$ are given in (4), and $\tilde{T}_{i}(Q)$ are the renormalized one-loop contributions at the scale $Q$. Here we neglected the one-loop corrections for $t_{3}$ since we are only interested in the value of $\mu$.

Using the procedure underlined above, the whole mass spectrum can be calculated as a function of the input parameters $m_{0}, m_{3 / 2}, \tan \beta, \operatorname{sign}(\mu), \epsilon_{3}$, and $m_{\nu_{\tau}}$. In Fig. 1 , we show a scatter plot of the mass spectrum as a function of the scalar mass $m_{0}$ for $m_{3 / 2}=32 \mathrm{TeV}, \tan \beta=5$, and $\mu<0$, varying $\epsilon_{3}$ and $m_{\nu_{\tau}}$ according to $10^{-5}<\epsilon_{3}<1 \mathrm{GeV}$ and $10^{-6}<m_{\nu_{\tau}}<1 \mathrm{eV}$. The widths of the scatter plots show that the spectrum exhibits a very small dependence on $\epsilon_{3}$ and $m_{\nu_{\tau}}$. Throughout this section we use this range for $\epsilon_{3}$ and $m_{\nu_{\tau}}$ in all figures.

We can see from this figure that, for $m_{0} \gtrsim 170 \mathrm{GeV}$, the LSP is the lightest neutralino $\tilde{\chi}_{1}^{0}$ with the lightest chargino $\tilde{\chi}_{1}^{+}$almost degenerated with it, as in $R_{P}$-conserving AMSB. Nevertheless, the LSP is the lightest stau $\tilde{\tau}_{1}^{-}$for $m_{0} \lesssim 170$ $\mathrm{GeV}$. This last region of parameter space is forbidden in 
$R_{P}$-conserving AMSB, but perfectly possible in AMSB$\mathrm{BRpV}$ since the stau is unstable, decaying into $R_{P}$-violating modes with sizeable branching ratios.

In our model, the $\mathrm{CP}-$ odd Higgs sector mixes with the imaginary part of the tau-sneutrino due to the bilinear $R_{P}$ violating interactions. Writing the mass terms in the form

$$
V_{\text {quadratic }}=\frac{1}{2}\left[\varphi_{d}^{0}, \varphi_{u}^{0}, \tilde{\nu}_{\tau}^{i 0}\right] M_{P^{0}}^{2}\left[\begin{array}{c}
\varphi_{d}^{0} \\
\varphi_{u}^{0} \\
\tilde{\nu}_{\tau}^{i 0}
\end{array}\right]
$$

we have

$$
\begin{gathered}
M_{P^{0}}^{2}= \\
{\left[\begin{array}{ccc}
m_{A}^{2(0)} s_{\beta}^{2}+\mu \epsilon_{3} \frac{v_{3}}{v_{d}} & m_{A}^{2(0)} s_{\beta} c_{\beta} & -\mu \epsilon_{3} \\
m_{A}^{2(0)} s_{\beta} c_{\beta} & m_{A}^{2(0)} c_{\beta}^{2}-\mu \epsilon_{3} \frac{v_{3}}{v_{d}} \frac{c_{\beta}^{2}}{s_{\beta}^{2}}+\frac{v_{3}^{2}}{v_{d}^{2}} \frac{c_{\beta}^{2}}{s_{\beta}^{2}} \bar{m}_{\tilde{\nu}_{\tau}}^{2} & -\mu \epsilon_{3} \frac{c_{\beta}}{s_{\beta}}+\frac{v_{3}}{v_{d}} \frac{c_{\beta}}{s_{\beta}} \bar{m}_{\tilde{\nu}_{\tau}}^{2} \\
-\mu \epsilon_{3} & -\mu \epsilon_{3} \frac{c_{\beta}}{s_{\beta}}+\frac{v_{3}}{v_{d}} \frac{c_{\beta}}{s_{\beta}} \bar{m}_{\tilde{\nu}_{\tau}}^{2} & \bar{m}_{\tilde{\nu}_{\tau}}^{2}
\end{array}\right],}
\end{gathered}
$$

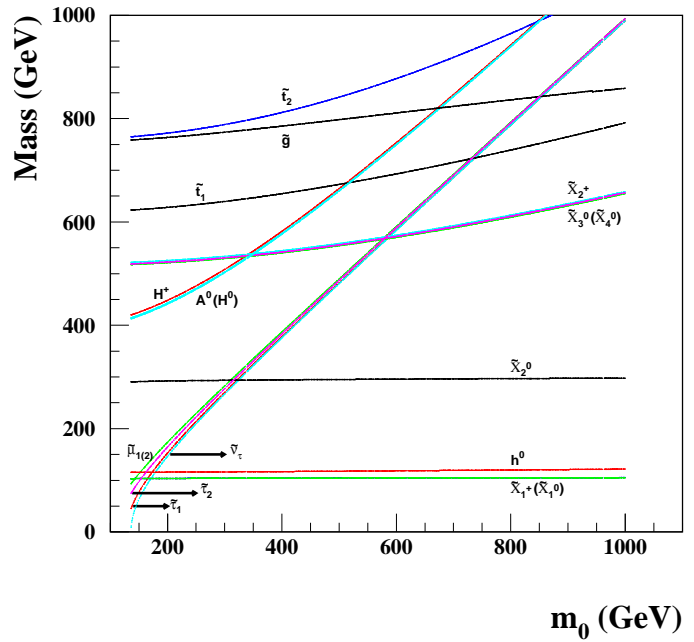

Figure 1. Supersymmetric mass spectrum in AMSB-BRpV for $m_{3 / 2}=32 \mathrm{TeV}, \tan \beta=5$, and $\mu<0$. The values of $\epsilon_{3}$ and $m_{\nu_{\tau}}$ were randomly varied according to $10^{-5}<\epsilon_{3}<1 \mathrm{GeV}$ and $10^{-6}<m_{\nu_{\tau}}<1 \mathrm{eV}$.

with $\bar{m}_{\tilde{\nu}_{\tau}}^{2}=m_{\tilde{\nu}_{\tau}}^{2(0)}+\epsilon_{3}^{2}+\frac{1}{8} g_{Z}^{2} v_{3}^{2}$ and $g_{Z}^{2} \equiv g^{2}+g^{\prime 2}$. Here,

$$
m_{A}^{2(0)}=\frac{B \mu}{s_{\beta} c_{\beta}} \text { and } m_{\tilde{\nu}_{\tau}}^{2(0)}=M_{L_{3}}^{2}+\frac{1}{8} g_{Z}^{2}\left(v_{d}^{2}-v_{u}^{2}\right)
$$

are respectively the $\mathrm{CP}$-odd Higgs and sneutrino masses in the $R_{P}$ conserving limit $\left(\epsilon_{3}=v_{3}=0\right)$. In order to write this mass matrix we have eliminated $m_{H_{u}}^{2}, m_{H_{d}}^{2}$, and $B_{3}$ using the tadpole equations (4). The mass matrix has an explicitly vanishing eigenvalue, which corresponds to the neutral Goldstone boson.

This matrix can be diagonalized with a rotation

$$
\left[\begin{array}{c}
A^{0} \\
G^{0} \\
\tilde{\nu}_{\tau}^{\text {odd }}
\end{array}\right]=R_{P^{0}}\left[\begin{array}{c}
\varphi_{d}^{0} \\
\varphi_{u}^{0} \\
\tilde{\nu}_{\tau}^{i 0}
\end{array}\right]
$$

where $G^{0}$ is the massless neutral Goldstone boson. Between the other two eigenstates, the one with largest $\tilde{\nu}_{\tau}^{i 0}$ component is called $\mathrm{CP}-$ odd tau-sneutrino $\tilde{\nu}_{\tau}^{\text {odd }}$ and the remaining state is called $\mathrm{CP}$-odd Higgs $A^{0}$.

As an intermediate step, it is convenient to make explicit the vanishing mass of the Goldstone boson with the rotation

$$
\widehat{R}_{P^{0}}=\left[\begin{array}{ccc}
s_{\beta} & c_{\beta} & 0 \\
-c_{\beta} r & s_{\beta} r & -\frac{v_{3}}{v_{d}} c_{\beta} r \\
-\frac{v_{3}}{v_{d}} c_{\beta}^{2} r & \frac{v_{3}}{v_{d}} s_{\beta} c_{\beta} r & r
\end{array}\right]
$$

where

$$
r=\frac{1}{\sqrt{1+\frac{v_{3}^{2}}{v_{d}^{2}} c_{\beta}^{2}}}
$$

obtaining a rotated mass matrix $\widehat{R}_{P^{0}} M_{P^{0}}^{2} \widehat{R}_{P^{0}}^{T}$ which has a column and a row of zeros, corresponding to $G^{0}$. This procedure simplifies the analysis since the remaining $2 \times 2$ mass matrix for $\left(A^{0}, \tilde{\nu}_{\tau}^{\text {odd }}\right)$ is

$$
\widehat{M}_{P^{0}}^{2}=\left[\begin{array}{cc}
m_{A}^{2(0)}+\frac{v_{3}^{2}}{v_{d}^{2}} \frac{c_{\beta}^{4}}{s_{\beta}^{2}} \bar{m}_{\tilde{\nu}_{\tau}}^{2}+\mu \epsilon_{3} \frac{v_{3}}{v_{d}} \frac{s_{\beta}^{2}-c_{\beta}^{2}}{s_{\beta}^{2}} & \left(\frac{v_{3}}{v_{d}} \frac{c_{\beta}^{2}}{s_{\beta}} \bar{m}_{\tilde{\nu}_{\tau}}^{2}-\mu \epsilon_{3} \frac{1}{s_{\beta}}\right) r \\
\left(\frac{v_{3}}{v_{d}} \frac{c_{\beta}^{2}}{s_{\beta}} \bar{m}_{\tilde{\nu}_{\tau}}^{2}-\mu \epsilon_{3} \frac{1}{s_{\beta}}\right) r & \bar{m}_{\tilde{\nu}_{\tau}}^{2} \frac{1}{r^{2}}
\end{array}\right]
$$



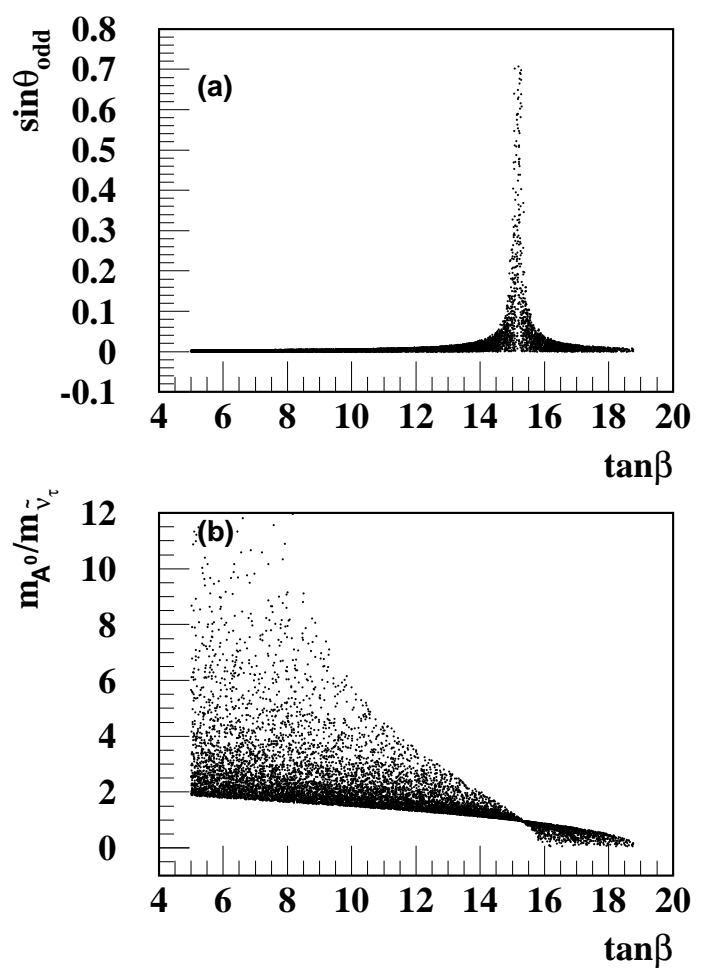

Figure 2. (a) $\mathrm{CP}$-odd Higgs-sneutrino mixing and (b) ratio between the $\mathrm{CP}$-odd Higgs mass and the sneutrino mass as a function of $\tan \beta$ for $m_{3 / 2}=32 \mathrm{TeV}, \mu<0$ and $100<m_{0}<300 \mathrm{GeV}$.

We quantify the mixing between the tau-sneutrino and the neutral Higgs bosons through

$$
\sin ^{2} \theta_{\text {odd }}=\left|\left\langle\tilde{\nu}_{\tau}^{\text {odd }} \mid \varphi_{u}^{0}\right\rangle\right|^{2}+\left|\left\langle\tilde{\nu}_{\tau}^{\text {odd }} \mid \varphi_{d}^{0}\right\rangle\right|^{2}
$$

If we consider the $R_{P}$ violating interactions as a perturbation, we can show that

$$
\sin ^{2} \theta_{\text {odd }} \simeq \frac{\left(\frac{v_{3}}{v_{d}} c_{\beta}^{2} m_{\tilde{\nu}_{\tau}}^{2(0)}-\mu \epsilon_{3}\right)^{2}}{s_{\beta}^{2}\left(m_{A}^{2(0)}-m_{\tilde{\nu}_{\tau}}^{2(0)}\right)^{2}}+\frac{v_{3}^{2}}{v_{d}^{2}} c_{\beta}^{2},
$$

indicating that this mixing can be large when the $\mathrm{CP}$-odd Higgs boson $A^{0}$ and the sneutrino $\tilde{\nu}_{\tau}$ are approximately degenerate.

Figure 2a displays the full sneutrino-Higgs mixing (13), with no approximations, as a function of $\tan \beta$ for $m_{3 / 2}=$ $32 \mathrm{TeV}, \mu<0$ and $100<m_{0}<300 \mathrm{GeV}$. In a large fraction of the parameter space this mixing is small, since it is proportional to the $\mathrm{BRpV}$ parameters squared divided by MSSM mass parameters squared. However, it is possible to find a region where the mixing is sizable, e.g., for our choice of parameters this happens at $\tan \beta \approx 15$. As expected, the region of large mixing is associated to near degenerate states, as we can see from Fig. $2 b$ where we present the ratio between the $\mathrm{CP}$-odd Higgs mass $m_{A}$ and the $\mathrm{CP}-$ odd tau-sneutrino mass $m_{\tilde{\nu}_{\tau}^{\text {odd }}}$ as a function of $\tan \beta$.

The CP-even Higgs/sneutrino sector and the Charged Higgs/charged slepton sector have similar behavior and their $R$-parity violating mixing angles can be as large as in the $\mathrm{CP}$-odd Higgs/sneutrino sector. For a more detailed analysis, see [17].

$\mathrm{BRpV}$ also provides a solution to the atmospheric and solar neutrino problems due to their mixing with neutralinos, which generates neutrino masses and mixing angles. It was shown in [6] that the atmospheric mass scale is adequately described by the tree level neutrino mass

$$
m_{\nu_{3}}^{\text {tree }}=\frac{M_{1} g^{2}+M_{2} g^{2}}{4 \Delta_{0}}|\vec{\Lambda}|^{2},
$$

where $\Delta_{0}$ is the determinant of the neutralino sub-matrix and $\vec{\Lambda}=\left(\Lambda_{1}, \Lambda_{2}, \Lambda_{3}\right)$, with

$$
\Lambda_{i}=\mu v_{i}+\epsilon_{i} v_{d}
$$

where the index $i$ refers to the lepton family. The spectrum generated is hierarchical, and obtained typically with $\Lambda_{1} \ll \Lambda_{2} \approx \Lambda_{3}$.

As it was mentioned before, for many purposes it is enough to work with $R_{P}$ violation only in the third generation. In this case, the atmospheric mass scale is well described by Eq. (15) with the replacement $|\vec{\Lambda}|^{2} \rightarrow \Lambda_{3}^{2}$. In Fig. , we plot the neutrino mass as a function of $\Lambda_{3}$ in AMSB-BRpV with the input parameters $m_{3 / 2}=32 \mathrm{TeV}$, $\mu<0,5<\tan \beta<20,100<m_{0}<1000 \mathrm{GeV}$ and $10^{-5}<\epsilon_{3}<1 \mathrm{GeV}$. The quadratic dependence of the neutrino mass on $\Lambda_{3}$ is apparent in this figure and neutrino masses smaller than $1 \mathrm{eV}$ occur for $\left|\Lambda_{3}\right| \lesssim 0.6 \mathrm{GeV}^{2}$. Moreover, the stars correspond to the allowed neutrino masses when the tau-sneutrino is the LSP. In general the points with a small (large) $m_{0}$ are located in the inner (outer) regions of this scattered plot.

From Fig. 3, we can see that the attainable neutrino masses are consistent with the global three-neutrino oscillation data analysis in [19] that favors the $\nu_{\tau} \rightarrow \nu_{\mu}$ oscillation hypothesis. At tree level, only one neutrino acquires a mass [20], which is proportional to the sneutrino vev in a basis where the bilinear $R$-parity violating terms are removed from the superpotential. At one-loop, three neutrinos get a non-zero mass, producing a hierarchical neutrino mass spectrum [15]. Although only mass squared differences are constrained by the neutrino data, our model naturally gives a hierarchical neutrino mass spectrum, therefore, we extract a naïve constraint on the actual mass coming from the analysis of the full atmospheric neutrino data, $0.04 \lesssim m_{\nu_{\tau}} \lesssim 0.09$ $\mathrm{eV}$ [19]. In addition, we notice that it is not possible to find an upper bound on the neutrino mass if angular dependence on the neutrino data is not included and only the total event rates are considered. 


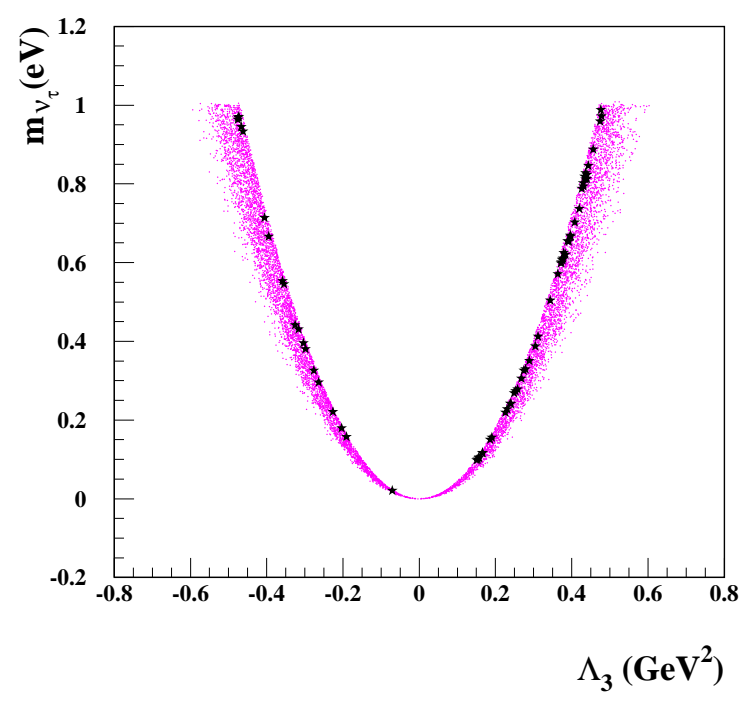

Figure 3. Tau neutrino mass as a function of $\Lambda_{3}$ for $5<\tan \beta<$ $20,100<m_{0}<1000 \mathrm{GeV}, m_{3 / 2}=32 \mathrm{TeV}$ and $\mu<0$. The stars correspond to the points where the tau-sneutrino is the LSP.

\section{Multilepton searches at the Teva- tron}

We also have searched for $R$-parity violating signals at the Run II of the Fermilab Tevatron in a scenario where supersymmetry is broken through SUGRA models. The parameter space of our SUGRA model, which exhibits $R$-parity violation only in the third generation, via the addition of the bilinear terms (1) and (2), is

$$
m_{0}, m_{1 / 2}, \tan \beta, \operatorname{sign}(\mu), A_{0}, \epsilon_{3} \text { and } m_{\nu_{\tau}},
$$

where the parameters $m_{1 / 2}$ and $m_{0}$ characterize the common gaugino mass and scalar soft SUSY breaking masses at the unification scale, $A_{0}$ is the common trilinear term, and $\tan \beta$ is the ratio between the Higgs field vev's. We characterize the BRpV sector by the $\epsilon_{3}$ term in the superpotential and the tau neutrino mass $m_{\nu_{\tau}}$ since it is convenient to trade the bilinear vev $v_{3}$ by $m_{\nu_{\tau}}$. In our simplified one-generation description considered here we fix, for definiteness, one representative value for $m_{\nu_{\tau}}=0.1 \mathrm{eV}$.

The presence of $\mathrm{BRpV}$ induces a mixing between the neutrinos and neutralinos, giving rise to the $R$-parity violating decays of the LSP. In our model, the lightest neutralino presents leptonic decays $\tilde{\chi}_{1}^{0} \rightarrow \nu \ell^{+} \ell^{-}$, semi-leptonic ones $\tilde{\chi}_{1}^{0} \rightarrow \nu q \bar{q}^{\prime}$ or $\ell q \bar{q}^{\prime}$, and the invisible mode $\tilde{\chi}_{1}^{0} \rightarrow \nu \nu \bar{\nu}$. The importance of the $\nu b \bar{b}$ decay mode increases for large $\epsilon_{3}$, since the effective coupling $\lambda_{333}^{\prime}$ is proportional to $\epsilon_{3}$ after a suitable rotation. For a fixed value of $\epsilon_{3}$, the branching ratio into $\nu b \bar{b}$ decreases with increasing $m_{\nu_{\tau}}$, as can be seen from Fig. 4. In general, the $\tilde{\chi}_{0}^{1}$ decays mainly into $\tau q \bar{q}^{\prime}$ for large $m_{0}$ or small $\epsilon_{3}$, while its decays are dominated by $\nu b \bar{b}$ at small $m_{0}$ and large $\epsilon_{3}$ and/or $\tan \beta$.
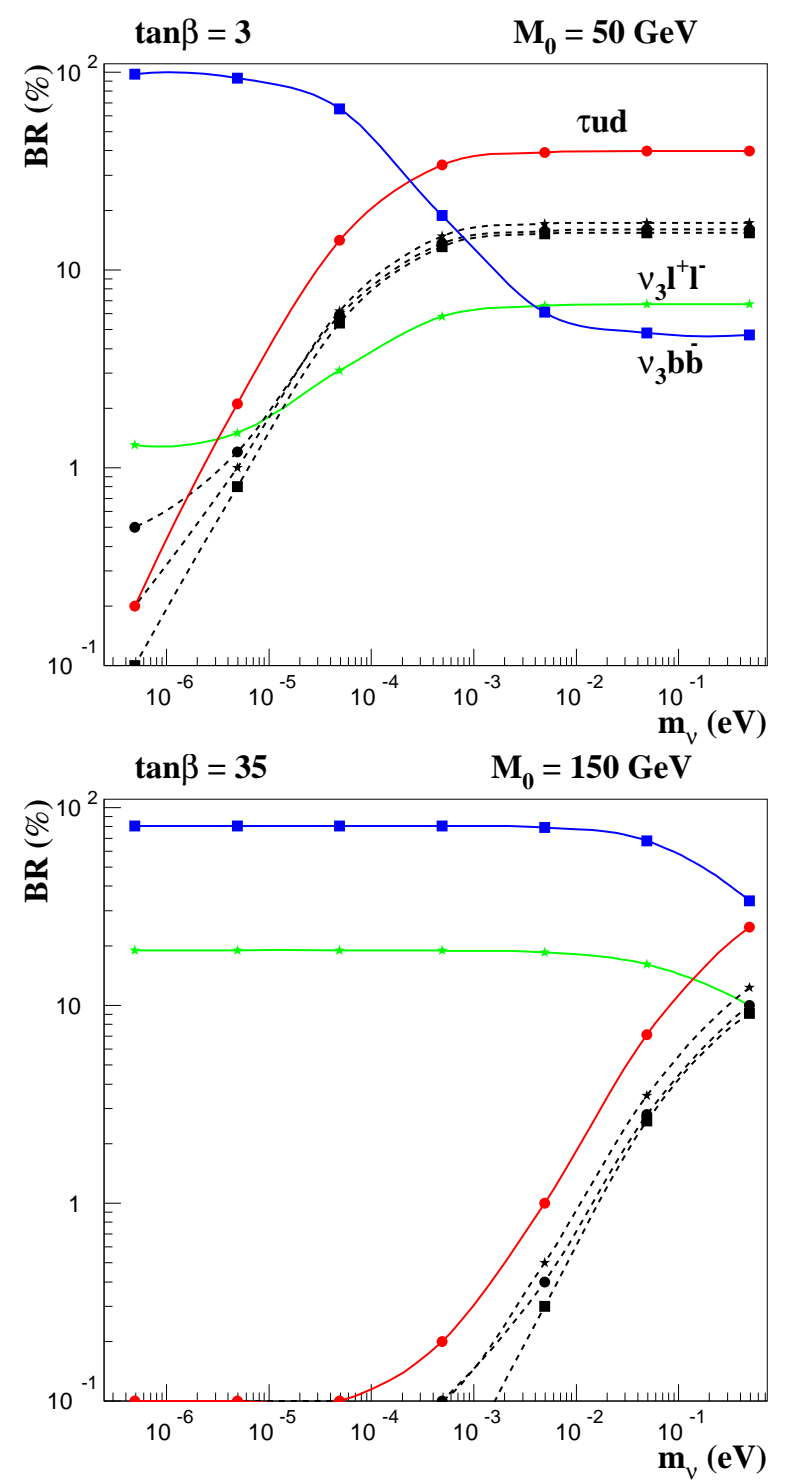

Figure 4. $\tilde{\chi}_{0}^{1}$ branching ratios as a function of $m_{\nu_{\tau}}$ for $A_{0}=0$, $\mu>0$, and $\epsilon_{3}=10^{-3} \mathrm{GeV}$. We fixed $m_{1 / 2}=175 \mathrm{GeV}$ in the case of $\tan \beta=3$ and $m_{1 / 2}=125 \mathrm{GeV}$ in the case of $\tan \beta=35$. The solid lines denote $\tilde{\chi}_{1}^{0} \rightarrow \nu_{3} b \bar{b}$ (squares); $\tilde{\chi}_{1}^{0} \rightarrow \tau \nu_{\ell} \ell$ (circles); and $\tilde{\chi}_{1}^{0} \rightarrow \nu_{3} \ell^{+} \ell^{-}$(stars). The dashed lines denote $\tilde{\chi}_{1}^{0} \rightarrow$ invisible (squares); $\tilde{\chi}_{1}^{0} \rightarrow \tau u d$ (circles); and $\tilde{\chi}_{1}^{0} \rightarrow \nu_{3} q \bar{q}$ (stars).

In $R$-parity conserving scenarios, the trilepton production at the Tevatron proceeds via $p \bar{p} \rightarrow \tilde{\chi}_{2}^{0} \tilde{\chi}_{1}^{ \pm}$with $\tilde{\chi}_{1}^{ \pm} \rightarrow$ $\ell \nu \tilde{\chi}_{1}^{0}, \tilde{\chi}_{2}^{0} \rightarrow \ell^{+} \ell^{-} \tilde{\chi}_{1}^{0}$, and the $\operatorname{LSP}\left(\tilde{\chi}_{1}^{0}\right)$ leaving the detector invisibly, producing then, 3 leptons in the final state. The main SM backgrounds for the trilepton production are displayed in Table 3. In order to suppress these backgrounds, we have imposed the soft cuts SC2 defined in Ref. [21], which were tailored for scenarios containing soft signal leptons coming from $\tau$ decays. In our analysis, the signal and backgrounds were generated using PYTHIA [22], except for the $W Z^{\star}\left(\gamma^{\star}\right)$ which was computed using the complete matrix elements. The trilepton cross section for the SM backgrounds after cuts are shown in Table 3.

As a good approximation, we have assumed that $\mathrm{BRpV}$ is only important for the $\tilde{\chi}_{0}^{1}$ decays and we incorporated these new modes in PYTHIA, leaving the other decays un- 
changed. In fact, the $R$-parity violating decay modes are strongly suppressed when the $R$ conserving ones have a few $\mathrm{GeV}$ of phase space. Assuming that gluinos and squarks are too heavy to be produced at the Tevatron, we considered the following processes: $p \bar{p} \rightarrow \tilde{\ell} \tilde{\ell}^{\star}, \tilde{\nu} \tilde{\ell}, \tilde{\chi}_{i}^{0} \tilde{\chi}_{j}^{0}(i(j)=1,2)$, $\tilde{\chi}_{1}^{+} \tilde{\chi}_{1}^{-}$, and $\tilde{\chi}_{i}^{0} \tilde{\chi}_{1}^{ \pm}(i=1,2)$.

The $\tilde{\chi}_{0}^{1}$ decays can contain charged leptons, and therefore, we should also analyze multilepton $(\geq 4 \ell)$ production. In order to extract this signal, we applied the trilepton case cuts but accepting leptons with $|\eta(\ell)|<3$. We also required the presence of an additional isolated lepton with $p_{T}>5 \mathrm{GeV}$ and demanded the missing transverse energy to be larger than $20 \mathrm{GeV}$. The main SM backgrounds for this process are $t \bar{t}, W Z$, and $Z Z$ productions whose cross sections after cuts are shown in Table I.

Table I. Background cross sections in $\mathrm{fb}$ for the trilepton and multilepton signatures at the Tevatron Run II.

\begin{tabular}{|l|c|c|}
\hline$\sigma(\mathrm{fb})$ & trilepton & multilepton \\
\hline \hline $\mathrm{WZ}(\mathrm{Z} \rightarrow \tau \tau)$ & 0.17 & 0.01 \\
\hline $\mathrm{W}^{*} \mathrm{Z}^{*}, \mathrm{~W}^{*} \gamma \rightarrow l l \bar{l}$ & 0.12 & - \\
\hline $\mathrm{W}^{*} \mathrm{Z}^{*}, \mathrm{~W}^{*} \gamma \rightarrow l l^{\prime} \bar{l}^{\prime}$ & 0.15 & - \\
\hline$t \bar{t}$ & 1.15 & 0.16 \\
\hline $\mathrm{Z}^{*} \mathrm{Z}^{*}$ & 0.05 & 0.10 \\
\hline total & 1.64 & 0.27 \\
\hline \hline
\end{tabular}

We investigated the regions of the $m_{0} \otimes m_{1 / 2}$ plane where the trilepton and multilepton signals can be established at the Tevatron for integrated luminosities of $2 \mathrm{fb}^{-1}$ and $25 \mathrm{fb}^{-1}$ and fixed values of $A_{0}, \tan \beta, \operatorname{sign}(\mu), \epsilon_{3}$, and $m_{\nu_{\tau}}$. We exhibit our results in the $m_{0} \otimes m_{1 / 2}$ plane, denoting by black circles the theoretically excluded points, and by white circles the experimentally excluded regions by sparticle and Higgs boson searches at LEP2 [23]. The black squares represent points accessible to Tevatron experiments at $5 \sigma$ level with $2 \mathrm{fb}^{-1}$ of integrated luminosity, while the white squares are accessible with $25 \mathrm{fb}^{-1}$. Points denoted by diamonds are accessible only at the $3 \sigma$ level with 25 $\mathrm{fb}^{-1}$, while the stars correspond to the region not accessible to the Tevatron. In the gray area, the $\tilde{\chi}_{0}^{1}$ decay length is rather long and this can modify our results, indicating that these points should be subject to a more detailed analysis. For more information on the decay lenght behavior see the full analysis at [24]. In Fig. 5, we present the region of the $m_{0} \otimes m_{1 / 2}$ plane that can probed at the Tevatron using the trilepton signal for $A_{0}=0, \tan \beta=3, \mu>0, \epsilon_{3}=10^{-3}$ $\mathrm{GeV}$, and $m_{\nu_{\tau}}=0.1 \mathrm{eV}$. For these values of the parameters, the $\tilde{\chi}_{0}^{1}$ decays mainly into $\tau q q^{\prime}$ and inside the detector for masses larger than $70 \mathrm{GeV}$.

It is interesting to compare our results presented in Fig. 5 with the ones in Ref. [21]. The presence of BRpV interactions reduces the Tevatron reach in the trilepton channel for small values of $m_{0}$. Here we have three competing effects: on the one side there are new contributions to the process, however, the decay of the neutralino into $\nu b \bar{b}$ produces a larger hadronic activity, destroying the lepton isolation, and reducing the missing $E_{T}$. Besides that, the leptons from the $\tilde{\chi}_{0}^{1}$ decay can give rise to additional isolated leptons which can contribute to the trilepton signal or suppress it due to the presence of more than three isolated leptons.

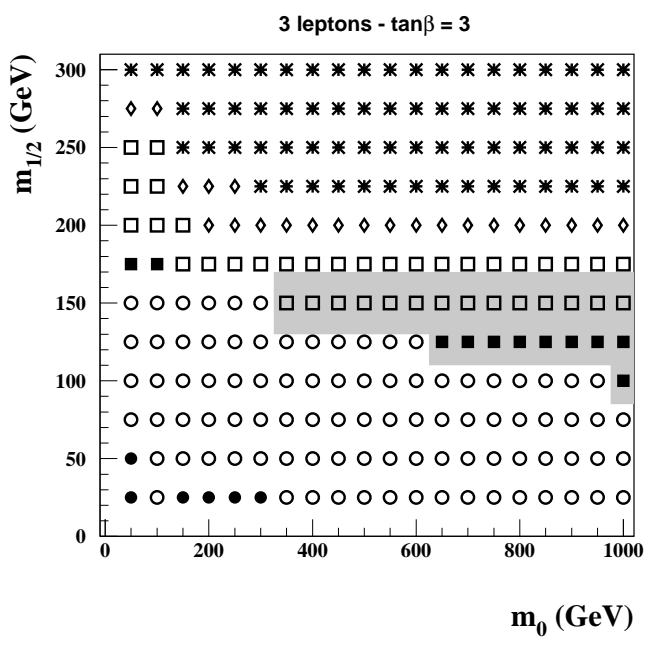

Figure 5. Reach of Fermilab Tevatron Run II using the trilepton signal in the $m_{0} \otimes m_{1 / 2}$ plane for $A_{0}=0, \tan \beta=3, \mu>0$, $\epsilon_{3}=10^{-3} \mathrm{GeV}$, and $m_{\nu_{\tau}}=0.1 \mathrm{eV}$. The black circles are theoretically excluded, while the white circles are experimentally excluded by sparticle and Higgs boson searches at LEP2. The black squares denote points accessible to Tevatron experiments at $5 \sigma$ level with 2 $\mathrm{fb}^{-1}$ of integrated luminosity, while the white squares are accessible with $25 \mathrm{fb}^{-1}$. Points denoted by diamonds are accessible at the $3 \sigma$ level with $25 \mathrm{fb}^{-1}$, while the stars correspond to the region not accessible to Tevatron. The grey area indicates the region where the neutralino has a large decay length, indicating that the results should be carefully revised.

We present the Tevatron reach in the multilepton channel in Fig. 6 for the same parameters adopted in Fig. 3. As we can see, the channel reach is larger than the trilepton one, increasing the discovery potential for larger values of $m_{1 / 2}$ or small $m_{0}$. In this region it is clear that the reduction of the trilepton signal is largely due to the presence of additional isolated leptons. We present the combined results for the trilepton and mulilepton searches in Fig. 7. It is interesting to notice that the presence of $R$-parity violating interactions leads to a $5 \sigma$ SUSY discovery even at large $m_{0}$, a region where the usual $R$-parity conserving SUGRA model has no discovery potential at all. Moreover, this result is only achieved by combining trilepton and multilepton signals. Nevertheless, a part of this result should be taken with care. For $m_{1 / 2} \lesssim 170 \mathrm{GeV}$ the lightest neutralino is lighter than $70 \mathrm{GeV}$ and has a large decay length for the parameters used in this analysis. Therefore, it is not guarateed that it will decay before the calorimeters. In principle, this could lead to spectacular events, which could increase the sensitivity to $\mathrm{BRpV}$, however, we consider them outside the scope of our analysis. In any case, it is clear that the presence of $\mathrm{BRpV}$ enhances the signal for $m_{1 / 2} \gtrsim 170 \mathrm{GeV}$ and large $m_{0}$. 


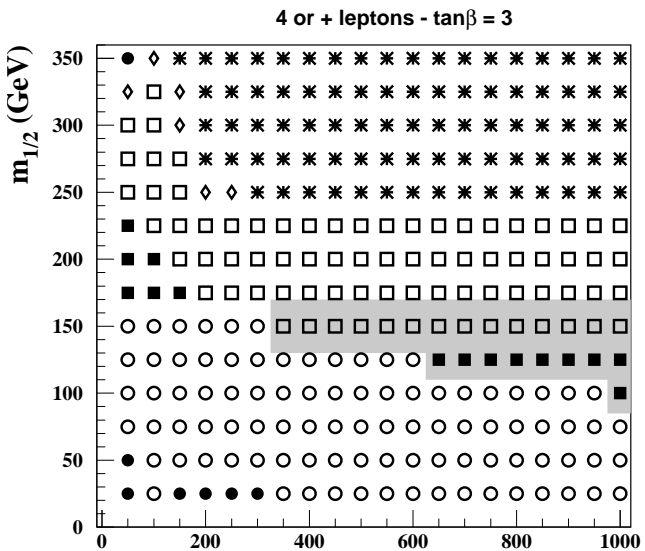

$\mathrm{m}_{0}(\mathrm{GeV})$

Figure 6. Reach of Fermilab Tevatron Run II in the 4 or more lepton channel. All parameters and conventions were chosen as in Fig. 5.

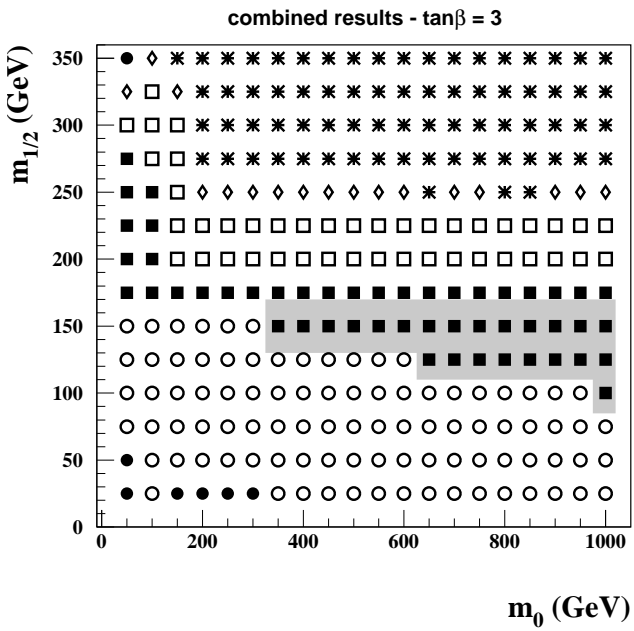

Figure 7. Reach of Fermilab Tevatron Run II combining trilepton and multilepton results. All parameters and conventions were chosen as in Fig. 5.

Finally, Fig. 8 displays the Tevatron reach for the combined channels for the case $A_{0}=0, \tan \beta=35, \mu>0$, $\epsilon_{3}=10^{-3} \mathrm{GeV}$ and $m_{\nu_{\tau}}=0.1 \mathrm{eV}$. For this choice of parameters, the main $\tilde{\chi}_{1}^{0}$ decay mode is $\tau q \bar{q}^{\prime}$, however, there is a sizeable contribution of the $\nu b \bar{b}$ channel at small $m_{0}$. As expected, the SUSY reach decreases at small $m_{0}$ as we increase $\tan \beta$, however there is a slight gain at large $m_{0}$. We also can see that the Tevatron reach diminishes when $\tan \beta$ is increased. Again, the gray area in the Fig. 8 shows the region where the LSP decay is rather long. We can see that for $\tan \beta=35$ this region is smaller and we should take with care the results in the region with $m_{1 / 2} \lesssim 140 \mathrm{GeV}$ and $m_{0} \gtrsim 190 \mathrm{GeV}$, where the lightest neutralino is lighter than $\sim 60 \mathrm{GeV}$.

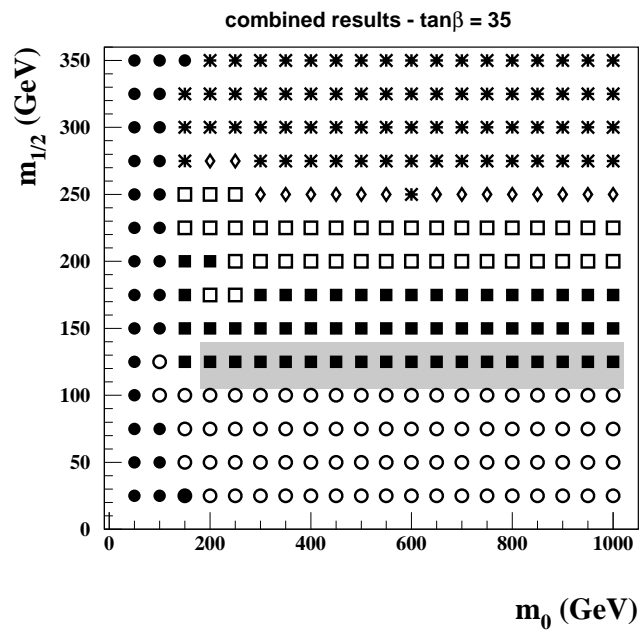

Figure 8. Reach of Fermilab Tevatron Run II combining trilepton and multilepton results for $A_{0}=0, \tan \beta=35, \mu>0$, $\epsilon_{3}=10^{-3} \mathrm{GeV}$ and $m_{\nu_{\tau}}=0.1 \mathrm{eV}$. The conventions are as in Fig. 5.

\section{Conclusions}

We have shown in the previous sections that our model exhibiting Anomaly Mediated Supersymmetry Breaking and Bilinear $R_{P}$ Violation is phenomenologically viable. In particular, the inclusion of BRpV generates neutrino masses and mixings in a natural way. Moreover, the $R_{P}$ breaking terms give rise to mixings between the Higgs bosons and the sleptons, which can be rather large despite the smallness of the parameters needed to generate realistic neutrino masses. These large mixings occur in regions of the parameter space where two states are nearly degenerate.

The $R_{P}$ violating interactions render the LSP unstable since it can decay via its mixing with the SM particles (leptons or scalars). Therefore, the constraints on the LSP are relaxed and forbidden regions of parameter space become allowed, where scalar particles like staus or sneutrinos are the LSP. Furthermore, the large mixing between Higgs bosons and sleptons has the potential to change the decays of these particles. These facts have a profound impact in the phenomenology of the model, changing drastically the signals at colliders [25].

We also have studied the trilepton and multilepton reach of the Tevatron in the simplest supergravity model where an effective bilinear term in the superpotential parametrizes the explicit breaking of $R$-parity. We have then shown how the presence of BRpV interactions leads to a suppression of the trilepton signal for small values of $m_{0}$ and/or large values of $\epsilon_{3}$ due to the $\tilde{\chi}_{0}^{1}$ decay into or $\tau q \bar{q}^{\prime}$ or $\nu b \bar{b}$. However, the $\tilde{\chi}_{0}^{1}$ decays lead to a drastically extended reach at large $m_{0}$, compensating the large hadronic decay of $\tilde{\chi}_{1}^{0}$. Moreover, the presence of additional isolated leptons in the signal allows us to look for multilepton events, specially important at large $m_{0}$. This new topology is useful not only for discovery, but also to verify whether $R$-parity is conserved or not. For a more detailed discussion on the results, see Ref. [24]. 
We demonstrated that combining the trilepton and multilepton searches increases the Tevatron Run II sensitivity for a large range of SUGRA and $R$-parity breaking parameters. It is interesting to notice that we can search for SUSY signals in the low $m_{0}$ region by looking for events exhibiting $b b b \ell$ or $b b b$ in association with missing transverse momentum [26]. Moreover, BRpV interactions lead to the production of extra $\tau$ leptons, therefore, it is possible to further increase the Tevatron reach for SUSY by allowing $\ell=\tau$ in our analyses since it is possible to detect $\tau$ pairs at the Tevatron [27].

In all of the above we have focused on the worst-case scenario, where we have only one generation and this is chosen to be the third. Our results are therefore robust, in the sense that the inclusion of additional generations would imply new sources of leptons, especially muons.

We would like to thank Fundação de Amparo à Pesquisa do Estado de São Paulo (FAPESP) for supporting this work.

\section{References}

[1] A. Cahmseddine, R. Arnowitt, and P. Nath, Phys. Rev. Lett. 49, 970 (1982); R. Barbieri, S. Ferrara, and C. Savoy, Phys. Lett. B119, 343 (1982); L. J. Hall, J. Lykken, and S. Weinberg, Phys. Rev. D27, 2359 (1983).

[2] M. Dine and A. Nelson, Phys. Rev. D48, 1277 (1993); M. Dine, A. Nelson, and Y. Shirman, Phys. Rev. D51, 1362 (1995); M. Dine, A. Nelson, Y. Nir, and Y. Shirman, Phys. Rev. D53, 2658 (1996).

[3] L. Randall and R. Sundrum, Nucl. Phys. B557, 79 (1999); G. Giudice, M. Luty, H. Murayama, and R. Ratazzi, JHEP 9812, 027 (1998).

[4] Q. R. Ahmad et al., [SNO Collaboration], Phys. Rev. Lett. 89, 011302 (2002); S. Fukuda et al., [Super-Kamiokande Collaboration], Phys. Lett. B539, 179 (2002); Phys. Rev. Lett. 81, 1562 (1998).

[5] C. S. Aulakh and R. N. Mohapatra, Phys. Lett. B119, 13 (1982); L. J. Hall and M. Suzuki, Nucl. Phys. B231, 41 (1984); G. G. Ross and J. W. Valle, Phys. Lett. B151, 375 (1985); J. R. Ellis et al., Phys. Lett. B150, 142 (1985).

[6] M. Hirsch et al., Phys. Rev. D62, 113008 (2000), [Erratumibid. D 65, 119901 (2002)]; J. C. Romão et al., Phys. Rev. D61, 071703 (2000).

[7] J. W. F. Valle, Phys. Lett. B199, 432 (1987).

[8] N. Fornengo, M. C. González-García, and J. W. F. Valle, Nucl. Phys. B580, 58 (2000); M. C. González-García, H.
Nunokawa, O. L. Perez, and J. W. F. Valle, Nucl. Phys. B543, 3 (1999); R. Foot, R. R. Volkas, and O. Yasuda, Phys. Rev. D58, 013006 (1998).

[9] M. C. González-García, P. C. de Holanda, C. Peña-Garay, and J. W. F. Valle, Nucl. Phys. B573, 3 (2000); S. Goswami, D. Majumdar, and A. Raychaudhuri, Phys. Rev. D63, 013003 (2001); J. N. Bahcall, P. I. Kratev, and A. Y. Smirnov, Phys. Rev. D62, 093004 (2000); G. L. Fogli, E. Lisi, D. Montanino, and A. Palazzo, Phys. Rev. D61, 073009 (2000).

[10] V. Barger and K. Whisnant, Phys. Rev. D59, 093007 (1999).

[11] M. Apollonio et al., Phys. Lett. B466, 415 (1999); F. Boehm et al., Phys. Rev. Lett. 84, 3764 (2000).

[12] V. Barger, S. Pakvasa, T. Weiler, and K. Whisnant, Phys. Lett. B437, 107 (1998); A. J. Baltz, A. S. Goldhaber, and M. Goldhaber, Phys. Rev. Lett. 81, 5730 (1998).

[13] A. Bartl et al., Nucl. Phys. B600, 39 (2001), and references therein.

[14] W. Porod, M. Hirsch, J. Romão, and J. W. F. Valle, Phys. Rev. D63, 115004 (2001), and references therein.

[15] M. A. Díaz, J. C. Romão, and J. W. Valle, Nucl. Phys. B524, 23 (1998).

[16] M. Maltoni, T. Scwetz, M. A. Tortola, and J. W. F. Valle, pre-print hep-ph/0207227.

[17] F. de Campos, M. A. Díaz, O. J. P. Éboli, M. B. Magro, and P. G. Mercadante, Nucl. Phys. B623, 47 (2002).

[18] T. Gherghetta, G. F. Giudice, and J. D. Wells, Nucl. Phys. B559, 27 (1999).

[19] M. C. González-García, M. Maltoni, C. Peña-Garay, and J. W. F. Valle, Phys. Rev. D63, 033005 (2001).

[20] A. Santamaria and J. W. F. Valle, Phys. Lett. B195, 423 (1987); Phys. Rev. Lett. 60, 397 (1988); Phys. Rev. D39, 1780 (1989).

[21] H. Baer, M. Drees, F. Paige, P. Quintana, and X. Tata, Phys. Rev. D 61, 095007 (2000).

[22] T. Söstrand, Computer Phys. Cammun. 82, 74 (1994).

[23] K. Hagiwara et al., Phys. Rev. D66, 010001 (2002).

[24] M. B. Magro et al., in preparation.

[25] F. de Campos et al., in preparation.

[26] V. D. Barger, T. Han, S. Hesselbach, and D. Marfatia, Phys. Lett. B538, 346 (2002).

[27] K. T. Matchev and D. M. Pierce, Phys. Rev. D 60, 075004 (1999). 